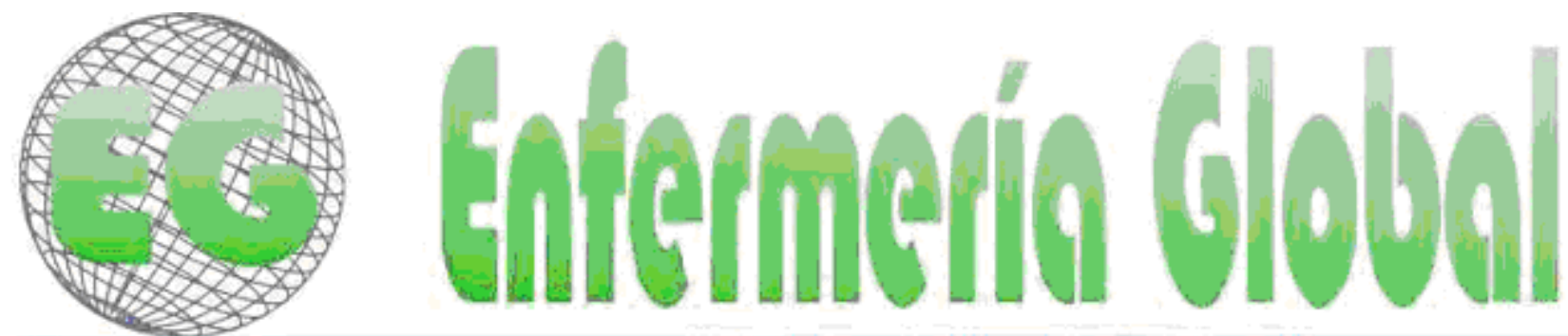

1SSN 1696-6*\$S

Revista electrônica cuatrimestral de Enfermeria

$\mathrm{N}^{\circ} 20$

Octubre 2010

www.um.es/egloball

REVISIONES

\title{
EL TRASTORNO LÍMITE DE PERSONALIDAD: LA FILOSOFÍA DIALÉCTICA COMO BASE DE LA INTERACCIÓN ENFERMERO PACIENTE
}

BORDERLINE PERSONALITY DISORDER: DIALECTIC PHILOSOPHY AS A BASE FOR NURSE-PATIENT INTERACTION

*Jiménez Barbero, JA., "*Pérez García, M., "**Rivera Rocamora, C., *Medina Garrido, L., **Munuera García, V., **Sánchez Muñoz, M.

"Enfermero/a. Unidad de Corta Estancia (UCE). Unidad de Hospitalización Psiquiátrica " Román Alberca" El Palmar. ${ }^{* *}$ Enfernera. Centro de Salud Mental de Lorca. ${ }^{* * *}$ Departamento de Enfermería. Universidad de Murcia.

Palabras clave: Enfermería, Trastorno límite de personalidad, Terapia, Cuidados.

Keywords: Nursing, Borderline Personality Disorder, Therapy, Treatment

\section{RESUMEN}

El trastorno límite de personalidad fue definido ya en el siglo XIX, como límite entre la psicosis y la neurosis. Presenta tres componentes clínicos principales: un inestable sentido del yo, un alto nivel de impulsividad y una elevada inestabilidad afectiva

Debido en parte, a la falta de conocimiento de la dinámica subyacente, el trabajo con pacientes con TLP resulta a menudo difícil para el personal de Enfermería.

El presente estudio tiene por objeto proponer orientaciones psicoterapéuticas que favorezcan la interacción en el cuidado de pacientes con este trastorno. Para este fin se lleva a cabo una búsqueda bibliográfica en las principales basos de datos de Enfermería y del resto de ciencias de la salud (Cochrane, Tripdatabase, Medline, CINAHL y CUIDEN), empleando los descriptores Mesh: "Borderline personality disorder" AND "therapy" y"Borderline personality" AND "nursing cares", indexados a partir del 01/01/2000, seleccionando finalmente un total de 17 estudios por su relevancia y relación con el tema.

Atendiendo a los resultados obtenidos, proponemos el empleo de la filosofía dialéctica, base de la terapia dialéctico-comportamental desarrollada por Marsha Linehan, como herramienta que va a conducir a una mejor relación enfermero-paciente, una mayor adherencia al tratamiento y una mayor eficiencia en la gestión de tiempo y recursos. 


\section{ABSTRACT}

Borderline personality disorder was defined in the nineteenth century as the boundary between psychosis and neurosis. It has three major clinical components: a shaky sense of self, a high level of impulsivity and high affective instability.

Due in part to the lack of knowledge of the underlying dynamics, working with patients with BPD is often difficult for nursing staff.

The present study aims to propose guidelines that encourage interactive psychotherapy in the care of patients with this disorder.

To this objective we conducted a bibliographic search in Nursing and other health science databases (Cochrane, Tripdatabase, Medline, CINAHL and care), using Mesh descriptors: "Borderline personality disorder" AND "therapy" and "Borderline Personality" AND "nursing cares", indexed from $01 / 01 / 2000$, finally selecting a total of 17 studies for their relevance and relationship to the subject under study.

Based on the results, we suggest the use of dialectical philosophy, the basis of dialectical-behavioral therapy developed by Marsha Linehan, a tool that will lead to a better nurse-patient relationship, greater adherence to treatment and greater efficiency in managing time and resources.

\section{INTRODUCCIÓN}

El trastorno límite de personalidad (TLP) se reconoció como enfermedad en el siglo XIX, en el límite entre la psicosis y la neurosis (Stone 1990). Las contribuciones psicoanalíticas posteriores reafirmaron esta distinción e hicieron énfasis en que su sentido de identidad es débil pero, no obstante, se conserva la capacidad para evaluar la realidad. De hecho, este trastorno parece presentar tres componentes clínicos principales. Primero, un inestable sentido del yo con dificultades en las relaciones interpersonales; segundo, la impulsividad, y tercero, la inestabilidad afectiva (1).

La prevalencia del trastorno de la personalidad borderline es aproximadamente del $2 \%$ en la población general, pero está presente en el $20 \%$ de los enfermos de psiquiatría hospitalizados y se diagnostica predominantemente en las mujeres (75\%) (2). Los problemas comunes coexistentes incluyen trastornos del estado de ánimo, abuso de sustancias, trastornos en los hábitos alimentarios y trastorno por estrés postraumático. El TLP también se asocia con otros trastornos de la personalidad y tiene una alta tasa de suicidio cuando se combina con trastornos del estado de ánimo o el alcoholismo (Stone 1990). El problema del daño autoinfligido y deliberado resulta también prevalente en este trastorno.

El trabajo con pacientes TLP a menudo resulta difícil para Enfermería, tal vez debido a la falta de comprensión de la dinámica subyacente de la enfermedad (3). De hecho, un interesante estudio de diseño cualitativo reveló tres áreas temáticas en la percepción que tienen los pacientes TLP del contacto con los profesionales de salud mental: la vida en el límite, la lucha por la salud y conservación de su dignidad, y las buenas y malas actuaciones de los profesionales de salud mental en el drama de su sufrimiento (4).

En este contexto nos parece esencial un adecuado abordaje por parte de Enfermería, teniendo en cuenta que el cuidado de pacientes con este diagnóstico ha sido identificado como un grave problema para los profesionales sanitarios. Investigaciones recientes, que sugieren que un diagnóstico de TLP influirá en el nivel y la calidad de la interacción personal con los pacientes (5), nos llevan a pensar que el cuidado de éstos constituye uno de los mayores desafíos a los que se enfrenta actualmente nuestra profesión.

El objetivo del presente estudio es proponer, basándonos en los resultados obtenidos por estudios enfermeros recientes, el empleo de la filosofía dialéctico-comportamental en la 
intervención de Enfermería, con el fin de ayudar a las personas con TLP a construir estrategias de afrontamiento eficaces y habilidades en respuestas conductuales para mejorar su calidad de vida (3).

\section{MATERIAL Y MÉTODO}

Con el fin de conocer las opciones terapéuticas que existen actualmente con relación al Trastono Límite de Personalidad, llevamos a cabo una revisión bibliográfica sobre el tema. La búsqueda se realiza en las principales bases de datos (Biblioteca Cochrane, Tripdatabase, Medline, Lilacs, CINAHL y CUIDEN), empleando los descriptores Mesh:

-"Borderline personality disorder" AND "therapy";

-"Borderline personality" AND "nursing cares".

Los parámetros de selección de los estudios son: revisiones sistemáticas, con y sin metaanálisis, ensayos clínicos aleatorizados (ECA'S), y no aleatorizados y estudios descriptivos, indexados en los últimos 10 años, lo que garantiza la actualidad de las conclusiones.

\section{RESULTADOS}

Finalmente seleccionamos 17 artículos, excluyendo a aquellos que no resultan relevantes para el objeto de estudio. Por parte de Enfermería, dada la escasez de publicaciones, seleccionamos 1 ECA, 2 estudios descriptivos y 2 cualitativos, indexados en CINAHL (ver Tabla 1).

Las opciones de terapéuticas en pacientes con un diagnóstico médico de TLP, incluyen:

-La farmacoterapia (especialmente los estabilizadores de ánimo, antipsicóticos atípicos e ISRS).

-Las intervenciones psicoterapéuticas, que se centran en aumentar la tolerancia al estrés, la regulación afectiva, el cambio de creencias distorsionadas, y la introducción de nuevas relaciones sociales y habilidades para resolver problemas (6). En base a los resultados obtenidos, destacamos la existencia, en la actualidad, de dos corrientes:

*Psicoterapia de orientación psicoanalítica.

*Psicoterapia de orientación cognitivo-conductual.

- Enfermería ha publicado poco al respecto. Sin embargo, los escasos estudios se centran en el empleo de los conceptos propios de la terapia dialéctico-comportamental como eje de la interacción enfermero-paciente. 


\begin{tabular}{|c|c|c|c|c|c|c|c|}
\hline & & Cochrane & MEDLINE & Lilacs & CINAHL & CUIDEN & Tripdatabas \\
\hline \multirow[t]{2}{*}{ Metaanálisis } & № & 2 & 1 & - & - & - & - \\
\hline & Ref & $(7,12)$ & (8) & & & & \\
\hline \multirow{2}{*}{$\begin{array}{l}\text { Revisiones } \\
\text { sistemáticas }\end{array}$} & № & 1 & - & - & $\begin{array}{lll}- & -\end{array}$ & - & - \\
\hline & Ref & (13) & & & & & \\
\hline \multirow[t]{2}{*}{ ECA'S } & № & - & 8 & - & 1 & $\begin{array}{lll}- & \\
-1\end{array}$ & - \\
\hline & Ref & & $\begin{array}{c}(9-11,15-17 \\
19,20)\end{array}$ & & (28) & & \\
\hline \multirow{2}{*}{\begin{tabular}{|l|} 
Estudios \\
descriptivos
\end{tabular}} & № & - & - & - & 2 & - & - \\
\hline & Ref & & & & $(5,29)$ & & \\
\hline \multirow{2}{*}{\begin{tabular}{|l|} 
Estudios \\
cualitativos
\end{tabular}} & № & - & - & - & 2 & - & - \\
\hline & Ref & & & & $(3,4)$ & & \\
\hline
\end{tabular}

\section{DISCUSIÓN}

\section{A) Tratamiento farmacológico}

El abordaje farmacológico de pacientes con TLP ha sido ampliamente estudiado desde diversos sectores (entre los que cabe destacar algunos vinculados a importantes laboratorios). Sin embargo, tras revisar lo publicado hasta la fecha, consideramos que aún no se han obtenido resultados que aporten evidencia científica, sobre la total efectividad de los mismos en la reducción de la sintomatología propia del trastorno límite de personalidad:

- El principal estudio al respecto lo constituye una revisión sistemática, que incluye un metaanálisis, indexada en la Biblioteca Cochrane en 2008 (7). Esta investigación, desarrollada por Binks y cols. incluye todos los ensayos clínicos aleatorios publicados hasta octubre de 2002, que comparaban cualquier fármaco psicoactivo con otro tratamiento para personas con TLP: se encontraron diez estudios aleatorios pequeños (total $n=554$ ) y cortos que incluían ocho comparaciones. Las conclusiones expuestas por sus autores indican que se podría considerar el efecto positivo de los antidepresivos en particular. Sin embargo, serían precisos ensayos bien diseñados, y con tamaños muestrales mayores que obtuvieran resultados clínicamente significativos.

- En 2006 Nosé M y cols., publican un metaanálisis en el que analizan los estudios que tratan de evaluar la eficacia del tratamiento farmacológico para los rasgos fundamentales del TLP (8). Seleccionan 22 ensayos clínicos aleatorizados y controlados con placebo, que proporcionan los siguientes resultados:

* Los antipsicóticos ejercen un efecto positivo respecto a la impulsividad, las relaciones interpersonales y el funcionamiento global. 
* Antidepresivos y estabilizadores de ánimo demuestran ser eficaces en el tratamiento de la inestabilidad afectiva y la ira, pero no frente a la impulsividad-agresión, la inestabilidad de las relaciones, las tendencias suicidas y el funcionamiento global.

- Asimismo, en un ECA desarrollado en España por Pascual JC y cols. durante el período de 2004 a 2006, que trata de evaluar la eficacia y tolerabilidad de la ziprasidona en el tratamiento de adultos diagnosticados con TLP no observa diferencias significativas entre el grupo experimental y el grupo control (9).

Aunque en algunos de los estudios analizados obtienen resultados positivos respecto al uso de psicofármacos, creemos que los mismos adolecen de tamaños muestrales suficientemente representativos, que los dote de una mayor validez externa. Este es el caso, por ejemplo, de sendos estudios realizado por Nickel MK y cols. en 2005 y 2006 respecto al topiramato $(10 ; 11)$.

En definitiva, vista la falta de evidencias de la eficacia del tratamiento farmacológico, el cuidado de los pacientes con TLP ha de pasar necesariamente por un abordaje basado principalmente en estrategias psicoterapéuticas.

\section{B) Tratamiento psicoterapéutico}

A este respecto, el estudio más importante hasta la fecha lo constituye sin duda una revisión sistemática que incluye un metaanálisis, también elaborada por Binks y cols., quienes plantean como objetivo principal evaluar los efectos de las intervenciones psicológicas para las personas con TLP (12). Los estudios incluidos en la misma fueron ECA'S relevantes que incluían tratamientos psicológicos para personas con TLP: tratamientos conductuales, cognitivo-conductuales, psicodinámicos y psicoanalíticos.

En base a este estudio, podemos decir que en la actualidad se desarrollan dos claras tendencias en el abordaje psicoterapéutico del Trastorno Límite:

1) Terapias de orientación psicoanalítica a las cuales pertenecen:

*Terapias psicodinámicas (TPD): en una reciente revisión sistemática, desarrollada por Abbass AA y cols., no encuentran ningún estudio de interés que evalúe la eficacia de este tratamiento en el TLP (13).

*El tratamiento basado en la mentalización (TBM), es una psicoterapia manualizada orientada psicodinámicamente para el TLP que tiene como objetivo desarrollar un proceso terapéutico en el que la capacidad del paciente para la mentalización se convierte en el foco del tratamiento (14). En este sentido destacamos el trabajo de Bateman y Fonagy, iniciado en 1999, en el que mediante un diseño experimental y aleatorio plantearon comparar el TBM de la hospitalización parcial orientada psicoanalíticamente con la atención psiquiátrica estándar (15). Los autores observan una disminución estadísticamente significativa de todas las medidas en contraste con el grupo control (atención psiquiátrica estándar), en la que los cambios fueron limitados o incluso se observó algún deterioro.

* Psicoterapia basada en la transferencia (PBT), una de las más empleadas en el tratamiento del TLP actualmente y defendida por Clarkin y Kernberg, que en un estudio realizado en 2006 concluyen que la PBT, tras 1 año de tratamiento, puede aumentar la coherencia narrativa y la función reflexiva de estos pacientes (16). 
El mismo grupo de investigación, en este caso coordinado por Clarkin, publicará en 2007 un ambicioso estudio multicéntrico en el que se comparan de nuevo tres tipos de tratamiento: la terapia dialéctico-comportamental (TDC), la psicoterapia basada en la transferencia (PBT) y el tratamiento de apoyo dinámico (TAD), (17): 90 pacientes, diagnosticados de TLP según criterios DSM-IV, son asignados al azar, a cada uno de los tres grupos de tratamiento descritos, durante 1 año. Los resultados obtenidos favorecen a las terapias de orientación psicoanalítica: aunque los tres tratamientos obtienen resultados positivos, los autores consideran que fue la PBT la que se asoció a mayor número de cambios.

\section{2) Terapias de orientación cognitivo-conductual, dentro de las cuales destacamos:}

*Terapia centrada en el esquema concebida por Young y que representa una ampliación del modelo de terapia cognitiva propuesta por Beck. Inicialmente fue presentada como una alternativa al tratamiento de los trastornos de personalidad, pero al igual que sucediera con la terapia cognitiva de Beck para la depresión, se ha utilizado en otros trastornos psicológicos.

Young plantea 18 esquemas desadaptativos tempranos que agrupa en las dimensiones: desconexión y rechazo, perjuicio en autonomía y desempeño, límites inadecuados, tendencia hacia el otro y sobrevigilancia e inhibición. Todos ellos se refieren a las situaciones que se establecen en el ambiente familiar(18).

*Terapia cognitivo-conductual (TCC), la cual parece ofrecer cambios clínicamente importantes en relativamente pocas sesiones clínicas reales, especialmente en el índice de peligro en un año, el estado de ansiedad, las creencias disfuncionales y la cantidad de actos suicidas, según diversos autores (19).

*Dejamos para el final la terapia dialectico-comportamental (TDC), la cual es un tratamiento psicosocial desarrollado por Marsha M. Linehan, a partir de la terapia cognitivoconductual estándar, específicamente para el tratamiento de personas con TLP.

Básicamente, la TDC sostiene que algunas personas, debido a ambientes familiares de invalidación durante su formación y a factores biológicos aún desconocidos, reaccionan anormalmente al estímulo emocional: su nivel de excitabilidad se eleva mucho más rápidamente, llega al máximo a un nivel más alto, y les toma más tiempo volver al punto de partida. Esto explica por qué las líneas límite se conocen por vidas consteladas de crisis e inestabilidad emocional extrema (emociones que cambian rápidamente). A causa de su invalidación pasada, no tienen ningún método para enfrentarse a estas oleadas repentinas e intensas de emoción. La TDC es un método que sirve para enseñarles habilidades que serán útiles para esta tarea (20).

El balance entre estrategias de cambio y de aceptación forma la dialéctica fundamental que da nombre al tratamiento. Dialéctica significa "evaluar e integrar ideas y hechos contradictorios en una visión que resuelve las contradicciones aparentes" (21).

Enfermero y paciente deben trabajar, según este modelo, para establecer un equilibrio entre el cambio (objetivo tradicional de la TCC) y la aceptación, dos fuerzas o estrategias aparentemente contradictorias: aceptación y cambio se convierten así en los conceptos claves que deben sustentar la actuación enfermera con los pacientes TLP: el enfermero deberá intentar guiar al paciente para que se acepte a sí mismo, a los demás y al mundo tal cual es. El abordaje dialéctico-comportamental de los pacientes con TLP desde Enfermería, tiene una sólida base teórica en el Modelo del Sistema Conductual propuesto por Dorothy Johnson, que define Enfermería como "una fuerza externa que actúa para preservar la organización conductual del paciente mientras éste se encuentra sometido a estrés, forzando sus mecanismos reguladores o bien proporcionándole recursos para ello" (22). 
Asimismo, según un reciente estudio enfermero (3), la TDC resulta de fácil implementación para Enfermería, favorece la gestión de los síntomas, y es rentable, en el sentido de que supone una más eficiente gestión del tiempo y de los recursos: en la típica configuración de la atención de salud mental, las enfermeras pueden aplicar eficazmente las intervenciones utilizando la filosofía dialéctica, ya sea en grupos o individualmente para ayudar a los clientes con TLP a lograr el bienestar óptimo.

Cabe añadir, que resulta indispensable en cualquier caso, pero aún más cuando prestamos cuidados a pacientes diagnosticados de TLP, la colaboración con el resto del equipo terapéutico para el seguimiento de los pacientes y el progreso del tratamiento. La actuación, por tanto, debe ser coordinada y la comunicación fluida y bidireccional entre el equipo terapéutico (integrado tradicionalmente por las Áreas de Psiquiatría, Psicología Clínica, Enfermería, Trabajo Social y Terapia Ocupacional) y el resto del personal sanitario; quizá ello conlleve replantear el concepto de equipo terapéutico, que debería integrar, no sólo a las Áreas mencionadas, sino también al resto de personal que pueda entrar en contacto (terapéutico o no), con estos pacientes: auxiliares de enfermería, celadores, e incluso en determinados casos, al personal de seguridad.

En nuestro caso, el Cuidado de Enfermería irá enfocado, desde la perspectiva planteada aquí, es decir, desde la filosofía dialéctica, a ayudar a los pacientes con TLP a construir estrategias de afrontamiento eficaces y aumentar su habilidad para generar respuestas conductuales adecuadas, elementos clave para mejorar su calidad de vida.

El presente estudio tiene, por lo tanto, el objetivo de proponer la intervención de Enfermería en el abordaje del Trastorno Límite de Personalidad, teniendo en cuenta las dificultades que supone a menudo la interacción con estos pacientes. Es por ello, que creemos que una intervención enfermera, planteada desde el PAE, y sustentada sobre las bases de la TDC, puede cobrar un especial protagonismo en una mejor evolución de esta enfermedad.

\section{C) Actuación de Enfermería}

-VALORACIÓN de Enfermería para el trastorno límite de personalidad o trastorno de personalidad borderline:

De acuerdo con el marco de valoración por patrones funcionales de salud, propuesto por Marjory Gordon (23), y teniendo en cuenta que los tres componentes clínicos principales del TLP son las dificultades en las relaciones interpersonales, la impulsividad, y la inestabilidad afectiva (1), los patrones que con más frecuencia van a aparecer de forma disfuncional en este tipo de pacientes son:

1.- Patrón Percepción y Manejo de la Salud. Este patrón hace referencia a la opinión de la persona sobre su salud y a la manera en que la entiende, controla y cuida (24). Tendríamos en cuenta en este caso los siguientes parámetros de registro: aspecto general, conocimiento sobre su problema, actitud y comportamientos frente al mismo y hábitos tóxicos, fundamentalmente

7.- Patrón Autopercepción-Autoconcepto. Describe las actitudes hacia uno mismo, la percepción de las capacidades cognitivas, afectivas y físicas, la imagen, la identidad, el sentido general de valía y el patrón emocional general, por lo que se encuentra frecuentemente alterado en este tipo de pacientes, con un yo inestable y en constante fluctuación. Valoraríamos su reactividad emocional, la percepción de su imagen corporal y actitud frente al mismo, y la percepción de exigencias y presiones a sí mismo. 
8.- Patrón Rol-Relaciones. También frecuentemente disfuncional en personas cuyo "yo" inestable, egocentrismo y dificultad para la empatía, les supone una gran dificultad para el establecimiento y/o conservación de las relaciones interpersonales.

9.- Patrón Sexualidad-Reproducción. Su alto nivel de impulsividad, unido a su inestabilidad afectivo-emocional y su alta tendencia al consumo de drogas, favorece en gran medida en estos pacientes, la manifestación de conductas sexuales de riesgo que pueden conllevar embarazos no deseados, ETS...

10.- Patrón de Adaptación-Tolerancia al estrés. Este patrón describe el nivel de estrés que la persona trata de forma efectiva. En el caso de pacientes que presentan dificultad para controlar sus impulsos nos interesará conocer:

-Cómo el individuo y la familia acepta, reacciona y se enfrenta a determinados problemas.

- La efectividad de los métodos de compensación.

-La concordancia entre la situación, el peligro percibido y el nivel de estrés (24).

DIAGNÓSTICOS de Enfermería para el trastorno límite de personalidad o trastorno de personalidad borderline (25)

Teniendo en cuenta que, como se ha dicho anteriormente, suelen ser problemas comunes coexistentes en el TLP los trastornos del estado de ánimo, el abuso de sustancias, y los trastornos en los hábitos alimentarios, y que además presenta una alta tasa de suicidio y/o daño autoinfligido y deliberado cuando se combina con trastornos del estado de ánimo o el alcoholismo (Stone 1990), lo diagnósticos de enfermería que vamos a encontrar con más frecuencia serían:

1.-Riesgo de violencia autodirigida [00140]

2.-Baja autoestima situacional [00120]

3.-Deterioro de la interacción social [00052]

4.-Conocimientos deficientes: enfermedad, tratamiento y evolución [00126]

Criterios de resultado (NOC) (26), indicadores (escalas de medición) e intervenciones (NIC) (27):

\section{DIAGNÓSTICO 1: "Riesgo de violencia autodirigida [00140]"}

-Definición: Riesgo de conductas que indiquen que una persona puede ser física, emocional o sexualmente lesiva para sí misma.

Resultados NOC e indicadores:

RESULTADO: Control de la automutilación [1406]

\begin{tabular}{|l|l|l|l|l|l|l|l|}
\hline CÓDIGOS & $\begin{array}{l}\text { INDICADORES } \\
\text { De Nunca Demostrado (N/D) a } \\
\text { Siempre Demostrado(S/D) }\end{array}$ & N/D & R/D & A/D & F/D & S/D & N/A \\
\hline 140602 & $\begin{array}{l}\text { Busca ayuda cuando siente la } \\
\text { necesidad } \\
\text { de autolesionarse }\end{array}$ & 1 & 2 & 3 & 4 & 5 & \\
\hline
\end{tabular}




\begin{tabular}{|l|l|l|l|l|l|l|l|}
\hline 140605 & $\begin{array}{l}\text { Mantiene el autocontrol sin } \\
\text { supervisión }\end{array}$ & 1 & 2 & 3 & 4 & 5 & \\
\hline 140606 & No se autolesiona & 1 & 2 & 3 & 4 & 5 & \\
\hline
\end{tabular}

RESULTADO: Autocontrol del impulso suicida [1408]

\begin{tabular}{|l|l|l|l|l|l|l|l|}
\hline CÓDIGOS & INDICADORES & N/D & R/D & A/D & F/D & S/D & N/A \\
\hline 140804 & $\begin{array}{l}\text { Verbaliza ideas de suicidio, si } \\
\text { existen }\end{array}$ & 1 & 2 & 3 & 4 & 5 & \\
\hline 140809 & $\begin{array}{l}\text { Se contiene de utilizar sustancias } \\
\text { modificadoras del estado de } \\
\text { ánimo }\end{array}$ & 1 & 2 & 3 & 4 & 5 & \\
\hline 140813 & $\begin{array}{l}\text { No intenta suicidarse intenta causarse lesiones } \\
\text { No } \\
\text { graves }\end{array}$ & 1 & 2 & 3 & 4 & 5 & \\
\hline
\end{tabular}

Intervenciones NIC para el diagnóstico Riesgo de violencia autodirigida [00140]:

-Control del humor [5330]: Proporcionar seguridad, estabilidad, recuperación y mantenimiento a un paciente que experimenta un humor disfuncionalmente deprimido o eufórico.

-Manejo de la conducta: autolesión [4354]: Ayuda al paciente para que disminuya o elimine la conducta de autolesión o abuso de sí mismo.

-Prevención del suicidio [6340]: Disminución del riesgo de los daños autoinfligidos con la intención de acabar con la vida.

-Manejo ambiental: seguridad [6486]: Vigilar y actuar sobre el ambiente físico para fomentar la seguridad.

\section{DIAGNÓSTICO 2: “Baja autoestima situacional [00120]"}

-Definición: Larga duración de una autoevaluación negativa o sentimientos negativos hacia uno mismo o sus capacidades.

Resultados NOC e indicadores:

RESULTADO: Autoestima [1205]

\begin{tabular}{|c|c|c|c|c|c|c|c|}
\hline CÓDIGOS & $\begin{array}{l}\text { INDICADORES: } \\
\text { De Nunca Positivo (N/P) a Siempre } \\
\text { Positivo (S/P) }\end{array}$ & N/P & $\mathrm{R} / \mathrm{P}$ & $A / P$ & $F / P$ & $\mathrm{~S} / \mathrm{P}$ & $\mathrm{N} / \mathrm{A}$ \\
\hline 120502 & $\begin{array}{l}\begin{array}{l}\text { Aceptación de las propias } \\
\text { limitaciones }\end{array} \\
\text { las }\end{array}$ & 1 & 2 & 3 & 4 & 5 & \\
\hline 120506 & Respeto por los demás & 1 & 2 & 3 & 4 & 5 & \\
\hline 120510 & $\begin{array}{l}\text { Equilibrio entre participar y } \\
\text { escuchar en grupos }\end{array}$ & 1 & 2 & 3 & 4 & 5 & \\
\hline
\end{tabular}




\begin{tabular}{|l|lrr|l|l|l|l|l|l|}
\hline 120514 & $\begin{array}{l}\text { Aceptación } \\
\text { constructivas }\end{array}$ & de & críticas & 1 & 2 & 3 & 4 & 5 & \\
\hline 120519 & $\begin{array}{l}\text { Sentimientos sobre su propia } \\
\text { persona }\end{array}$ & 1 & 2 & 3 & 4 & 5 & \\
\hline
\end{tabular}

RESULTADO: Modificación psicosocial: cambio de vida [1305]

\begin{tabular}{|c|c|c|c|c|c|c|c|}
\hline CÓDIGOS & $\begin{array}{l}\text { INDICADORES } \\
\text { De Nunca Demostrado } \\
\text { Siempre Demostrado (S/D) a }\end{array}$ & N/D & $R / D$ & $A / D$ & F/D & S/D & N/A \\
\hline 130501 & $\begin{array}{l}\text { Establecimiento de objetivos } \\
\text { realistas }\end{array}$ & 1 & 2 & 3 & 4 & 5 & \\
\hline 130502 & Mantenimiento de la autoestima & 1 & 2 & 3 & 4 & 5 & \\
\hline 130504 & Expresiones de utilidad & 1 & 2 & 3 & 4 & 5 & \\
\hline 130505 & $\begin{array}{l}\text { Expresiones de optimismo sobre } \\
\text { el presente }\end{array}$ & 1 & 2 & 3 & 4 & 5 & \\
\hline 130506 & $\begin{array}{l}\text { Expresiones de optimismo sobre } \\
\text { el futuro }\end{array}$ & 1 & 2 & 3 & 4 & 5 & \\
\hline 130507 & $\begin{array}{l}\text { Expresiones de sentimientos } \\
\text { permitidos }\end{array}$ & 1 & 2 & 3 & 4 & 5 & \\
\hline 130509 & $\begin{array}{l}\text { Uso de estrategias de superación } \\
\text { efectivas }\end{array}$ & 1 & 2 & 3 & 4 & 5 & \\
\hline
\end{tabular}

RESULTADO: Equilibrio emocional [1204]

\begin{tabular}{|c|c|c|c|c|c|c|c|}
\hline CÓDIGOS & INDICADORES & N/D & $\mathrm{R} / \mathrm{D}$ & $\mathrm{A} / \mathrm{D}$ & F/D & $S / D$ & N/A \\
\hline 120401 & Muestra un afecto apropiado & 1 & 2 & 3 & 4 & 5 & \\
\hline 120402 & $\begin{array}{l}\text { Muestra un estado de ánimo } \\
\text { sereno }\end{array}$ & 1 & 2 & 3 & 4 & 5 & \\
\hline 120403: & Muestra control de los impulsos & 1 & 2 & 3 & 4 & 5 & \\
\hline 120415 & $\begin{array}{l}\text { Muestra interés por lo que le } \\
\text { rodea }\end{array}$ & 1 & 2 & 3 & 4 & 5 & \\
\hline 120417 & $\begin{array}{l}\text { Refiere un nivel de energía } \\
\text { apropiado }\end{array}$ & 1 & 2 & 3 & 4 & 5 & \\
\hline 120418 & $\begin{array}{l}\text { Refiere capacidad para realizar } \\
\text { las tareas }\end{array}$ & 1 & 2 & 3 & 4 & 5 & \\
\hline
\end{tabular}

Intervenciones NIC para el diagnóstico Baja autoestima situacional [00120]:

-Potenciación de la socialización [5100]: Facilitar la capacidad de una persona para interactuar con otros.

-Potenciación de la autoestima [5400]: Ayudar a un paciente a que aumente el juicio personal de su propia valía. 


\section{DIAGNÓSTICO 3: "Deterioro de la interacción social [00052]"}

Definición: Intercambio social inefectivo o cuantitativamente insuficiente o excesivo.

Resultados NOC e indicadores:

RESULTADO: Implicación social [1503]

\begin{tabular}{|c|c|c|c|c|c|c|c|}
\hline CÓDIGOS & $\begin{array}{l}\text { INDICADORES } \\
\text { De Nunca Demostrado } \\
\text { Siempre Demostrado }(S / D)\end{array}$ & N/D & $R / D$ & $A / D$ & F/D & S/D & N/A \\
\hline 150301 & Interacción con amigos íntimos & 1 & 2 & 3 & 4 & 5 & \\
\hline 150303 & $\begin{array}{l}\text { Interacción con miembros de la } \\
\text { familia }\end{array}$ & 1 & 2 & 3 & 4 & 5 & \\
\hline 150311 & $\begin{array}{l}\text { Participación en actividades de } \\
\text { ocio }\end{array}$ & 1 & 2 & 3 & 4 & 5 & \\
\hline 150312 & $\begin{array}{|lll|}\begin{array}{l}\text { Interacción } \\
\text { sanitario }\end{array} & \text { con } & \text { personal } \\
\end{array}$ & 1 & 2 & 3 & 4 & 5 & \\
\hline 150314 & Interacción con otros pacientes & 1 & 2 & 3 & 4 & 5 & \\
\hline
\end{tabular}

RESULTADO: Severidad de la soledad [1203]

\begin{tabular}{|c|c|c|c|c|c|c|c|}
\hline CÓDIGOS & $\begin{array}{l}\text { INDICADORES } \\
\text { De Grave a Ninguno } \\
\text { moderado, leve, ninguno) }\end{array}$ & $\mathrm{G}$ & $S$ & $\mathbf{M}$ & L & $\mathrm{N}$ & N/A \\
\hline 120307 & Expresión de aislamiento social & 1 & 2 & 3 & 4 & 5 & \\
\hline 120312 & $\begin{array}{l}\text { Dificultad para establecer contacto con otras } \\
\text { personas }\end{array}$ & 1 & 2 & 3 & 4 & 5 & \\
\hline 120314 & $\begin{array}{l}\text { Dificultad para tener una relación mutua } \\
\text { eficaz }\end{array}$ & 1 & 2 & 3 & 4 & 5 & \\
\hline 120315 & $\begin{array}{l}\text { Demostración de fluctuaciones en el estado } \\
\text { de ánimo }\end{array}$ & 1 & 2 & 3 & 4 & 5 & \\
\hline
\end{tabular}

RESULTADO: Soporte social [1504]

\begin{tabular}{|c|c|c|c|c|c|c|c|c|}
\hline CÓDIGOS & $\begin{array}{l}\text { INDICADORES } \\
\text { De Inadecuado } \\
\text { Adecuado }\end{array}$ & Completamente & 1 & $\begin{array}{l}\text { L/ } \\
\mathrm{A}\end{array}$ & $\mathrm{M} / \mathrm{A}$ & S/A & $\mathrm{C} / \mathrm{A}$ & N/A \\
\hline 150406 & Refiere relaciones & confianza & 1 & 2 & 3 & 4 & 5 & \\
\hline 150410 & $\begin{array}{l}\text { Refiere contacto } \\
\text { soporte adecuados }\end{array}$ & sociales de & 1 & 2 & 3 & 4 & 5 & \\
\hline 150411 & Refiere una red so & estable & 1 & 2 & 3 & 4 & 5 & \\
\hline
\end{tabular}

Intervenciones NIC para el diagnóstico Deterioro de la interacción social [00052]:

-Modificación de la conducta: habilidades sociales [4362]: Ayudar al paciente para que desarrolle o mejore las habilidades sociales interpersonales. 
-Terapia de grupo [5450]: Aplicación de técnicas psicoterapéuticas a un grupo, incluyendo la utilización de interacciones entre los miembros del grupo.

-Potenciación de la socialización [5100]: Facilitar la capacidad de una persona para interactuar con otros.

\section{DIAGNÓSTICO 4: "Conocimientos deficientes: enfermedad, tratamiento y evolución [00126]"}

-Definición: Carencia o deficiencia de información cognitiva relacionada con su enfermedad, tratamiento y evolución.

La necesidad de intervenir sobre este diagnóstico enfermero, la fundamentamos en diversos estudios que avalan la importancia de la educación sobre su enfermedad en estos pacientes. Es el caso de un ensayo clínico aleatorizado, llevado a cabo por profesionales de enfermería, cuyo propósito fue evaluar el impacto de la educación clínica en pacientes con trastorno límite de la personalidad, en las actitudes de los médicos que trabajan con pacientes que han exhibido comportamientos autolesivos deliberados, propios de personas diagnosticadas con este trastorno (28).

\section{Resultados NOC e indicadores:}

\section{RESULTADO: Conocimiento: medicación [1808]}

\begin{tabular}{|c|c|c|c|c|c|c|c|}
\hline CÓDIGOS & $\begin{array}{l}\text { INDICADORES: } \\
\text { De Ninguno a Extenso (ninguno, escaso, moderado, } \\
\text { sustancial y extenso) }\end{array}$ & $\mathbf{N}$ & E & M & S & E & $\mathrm{N} / \mathrm{A}$ \\
\hline 180804 & Descripción de las acciones de la medicación & 1 & 2 & 3 & 4 & 5 & \\
\hline 180805 & $\begin{array}{l}\text { Descripción de los efectos indeseables de la } \\
\text { medicación }\end{array}$ & 1 & 2 & 3 & 4 & 5 & \\
\hline 180806 & $\begin{array}{l}\text { Descripción de las precauciones de la } \\
\text { medicación }\end{array}$ & 1 & 2 & 3 & 4 & 5 & \\
\hline 180810 & $\begin{array}{l}\text { Descripción de la administración correcta de la } \\
\text { medicación }\end{array}$ & 1 & 2 & 3 & 4 & 5 & \\
\hline
\end{tabular}

RESULTADO: Conocimiento: proceso de la enfermedad [1803]

\begin{tabular}{|c|c|c|c|c|c|c|c|}
\hline CÓDIGOS & INDICADORES & $\mathbf{N}$ & $E$ & M & $\mathrm{S}$ & $\bar{E}$ & N/A \\
\hline 180302 & Descripción del proceso de enfermedad & 1 & 2 & 3 & 4 & 5 & \\
\hline 180303 & $\begin{array}{l}\text { Descripción de la causa o factores } \\
\text { contribuyentes }\end{array}$ & 1 & 2 & 3 & 4 & 5 & \\
\hline 180304 & Descripción de los factores de riesgo & 1 & 2 & 3 & 4 & 5 & \\
\hline 180305 & Descripción de los efectos de la enfermedad & 1 & 2 & 3 & 4 & 5 & \\
\hline 180306 & Descripción de signos y síntomas & 1 & 2 & 3 & 4 & 5 & \\
\hline 180307 & $\begin{array}{l}\text { Descripción del curso habitual de la } \\
\text { enfermedad }\end{array}$ & 1 & 2 & 3 & 4 & 5 & \\
\hline
\end{tabular}


RESULTADO: Conocimiento: recursos sanitarios [1806]

\begin{tabular}{|l|l|l|l|l|l|l|l|}
\hline CÓDIGOS & INDICADORES & N & E & M & S & E & N/A \\
\hline 180602 & $\begin{array}{l}\text { Descripción de cuándo contactar con } \\
\text { profesionales sanitarios }\end{array}$ & 1 & 2 & 3 & 4 & 5 & \\
\hline $\begin{array}{l}\text { Descripción de la necesidad de la asistencia } \\
\text { continuada }\end{array}$ & 1 & 2 & 3 & 4 & 5 & \\
\hline 180605 & $\begin{array}{l}\text { Descripción de los recursos sociales de } \\
\text { ayuda disponibles }\end{array}$ & 1 & 2 & 3 & 4 & 5 & \\
\hline
\end{tabular}

RESULTADO: Conocimiento: régimen terapéutico [1813]

\begin{tabular}{|c|c|c|c|c|c|c|c|}
\hline CÓDIGOS & INDICADORES & $\mathbf{N}$ & $E$ & M & $S$ & $E$ & N/A \\
\hline 181301 & $\begin{array}{l}\text { Descripción de la justificación del régimen } \\
\text { terapéutico }\end{array}$ & 1 & 2 & 3 & 4 & 5 & \\
\hline 181302 & $\begin{array}{l}\text { Descripción de las responsabilidades de los } \\
\text { propios cuidados para el tratamiento actual }\end{array}$ & 1 & 2 & 3 & 4 & 5 & \\
\hline 181306 & Descripción de la medicación prescrita & 1 & 2 & 3 & 4 & 5 & \\
\hline 181316 & $\begin{array}{l}\text { Descripción de los beneficios del } \\
\text { tratamiento de la enfermedad }\end{array}$ & 1 & 2 & 3 & 4 & 5 & \\
\hline
\end{tabular}

Intervenciones NIC para el diagnóstico Conocimientos deficientes relacionados con su enfermedad, tratamiento y evolución [00126]:

-Potenciación de la disposición de aprendizaje [5540]: Mejorar la capacidad y disposición de recibir información.

-Enseñanza: medicamentos prescritos [5616]: Preparación de un paciente para que tome de forma segura los medicamentos prescritos y observar sus efectos.

-Enseñanza: proceso de la enfermedad [5602]: Ayudar al paciente a comprender la información relacionada con su proceso de enfermedad (en este caso con las manifestaciones típicas del TLP.

\section{CONCLUSIONES}

-Los estudios relativos al TLP, hasta el momento, nos indican que el tratamiento de este complejo trastorno requiere un abordaje multidisciplinar y multidimensional. Según los datos de que disponemos, a diferencia de lo que ocurre con otros trastornos mentales, como los de naturaleza psicótica, el tratamiento farmacológico del TLP no parece obtener resultados totalmente significativos, para una evolución positiva del mismo. Sin embargo, sí parece constituir un apoyo esencial para otro tipo de terapias más determinantes, como las psicodinámicas, la psicoterapia centrada en la transferencia o la terapia dialécticocomportamental.

-En este contexto, Enfermería se encuentra, en muchas ocasiones, en una posición difícil a la hora de abordar a estos pacientes: los enfermeros de salud mental se suelen quejar, frecuentemente, de la falta de resultados positivos con los actuales regímenes de atención. 
De hecho, un estudio cualitativo realizado en China entre enfermeros de salud mental que atienden a estos pacientes, ha obtenido resultados altamente insatisfactorios en este sentido. A pesar de ello, según las conclusiones del estudio, los pacientes podrían obtener mejores resultados por enfermeros con "actitudes positivas, dirigidas hacia la promoción de la salud de estos pacientes y contando con la recepción oportuna y el apoyo constante de los miembros del equipo de salud" (29).

-Consideramos que la utilización de los conceptos propios de la filosofía dialéctica (aceptación y cambio) podría constituir una herramienta eficaz para la prestación de cuidados enfermeros a estos pacientes, tanto en las intervenciones individuales (manejo de conductas proyectivas, gestión de crisis...), como en las grupales (programas psicoeducativos).

-En este modelo, enfermeros y pacientes deben trabajan arduamente para balancear cambio y aceptación, dos fuerzas o estrategias aparentemente contradictorias. Ello nos debe conducir a una mejor relación enfermero-paciente, una mayor adherencia al tratamiento y una mayor eficiencia en la gestión de tiempo y recursos.

-Puede ser especialmente relevante en estos casos, la figura del enfermero Especialista en Salud Mental, quien puede supervisar al resto del personal de enfermería y proporcionar educación al paciente acerca de la dinámica, las respuestas del personal y las decisiones del equipo terapéutico. Asimismo puede ofrecer apoyo emocional al resto del personal de Enfermería, lo cual conducirá a un aumento en su satisfacción en el trabajo, considerando la dificultad que existe para mantener la conducta terapéutica profesional con estos pacientes (30).

\section{REFERENCIAS}

(1) Critchfield KL, Levy KN, Clarkin JF. The relationship between impulsivity, aggression, and impulsive-aggression in borderline personality disorder: an empirical analysis of self-report measures. J Pers Disord 2004;18(6):555-70.

(2) Frances A, Pincus HA, First MB, Andreasen NC, Barlow DH, Campbell M. DSM-IVTR: Manual diagnóstico y estadístico de los trastornos mentales. Editorial Masson. $4^{\mathrm{a}}$ ed. 2002.

(3) Osborne LL, McComish JF. Working with borderline personality disorder: nursing interventions using dialectical behavioral therapy. J Psychosoc Nurs Ment Health 2006;44(6):40-9.

(4) Perseius K, Ekdahl S, Åsberg M, Samuelsson M. To tame a volcano: patients with borderline personality disorder and their perceptions of suffering. Arch Psychiatr Nurs 2005;19(4):160-8.

(5) Deans C, Meocevic E. Attitudes of registered psychiatric nurses towards patients diagnosed with borderline personality disorder. CONTEMP NURSE 2006;21(1):43-9.

(6) Hunt M. Borderline personality disorder across the life span. J WOMEN AGING 2007;19(1-2):173-91. 
(7) Binks CA, Fenton M, McCarthy L, Lee T, Adams CE, Duggan C. Intervenciones farmacológicas para las personas con trastorno de la personalidad borderline. La Biblioteca Cochrane Plus 2008;2.

(8) Nosè M, Cipriani A, Biancosino B, Grassi L, Barbui C. Efficacy of pharmacotherapy against core traits of borderline personality disorder: meta-analysis of randomized controlled trials. Int Clin Psychopharmacol 2006;21(6):345-53.

(9) Pascual JC, Soler J, Puigdemont D, Pérez-Egea R, Tiana T, Alvarez E, et al. Ziprasidone in the treatment of borderline personality disorder: a double-blind, placebo-controlled, randomized study. J Clin Psychiatry 2008;69(4):603-8.

(10) Nickel MK, Nickel C, Mitterlehner FO, Tritt K, Lahmann C, Leiberich PK, et al. Topiramate treatment of aggression in female borderline personality disorder patients: a double-blind, placebo-controlled study. J Clin Psychiatry 2004;65(11):1515-9.

(11) Nickel MK, Nickel C, Kaplan P, Lahmann C, Mühlbacher M, Tritt K, et al. Treatment of aggression with topiramate in male borderline patients: a double-blind, placebocontrolled study. Biol Psychiatry 2005;57(5):495-9.

(12) Binks CA, Fenton M, McCarthy L, Lee T, Adams CE, Duggan C. Terapias psicológicas para personas con trastorno de la personalidad borderline. La Biblioteca Cochrane Plus 2008;2.

(13) Abbass AA, Hancock JT, Henderson J, Kisely S. Psicoterapias psicodinámicas a corto plazo para trastornos mentales frecuentes. La Biblioteca Cochrane Plus 2008;2.

(14) Eizirik M, Fonagy P. Mentalization-based treatment for patients with borderline personality disorder: an overview. Rev Bras Psiquiatr 2009;31(1):72-5.

(15) Bateman A, Fonagy P. Effectiveness of partial hospitalization in the treatment of borderline personality disorder: a randomized controlled trial. Am J Psychiatry 1999;156(10):1563-9.

(16) Levy KN, Meehan KB, Kelly KM, Reynoso JS, Weber M, Clarkin JF, et al. Change in attachment patterns and reflective function in a randomized control trial of transference-focused psychotherapy for borderline personality disorder. J Consult Clin Psychol 2006;74(6):1027-40.

(17) Clarkin JF, Levy KN, Lenzenweger MF, Kernberg OF. Evaluating three treatments for borderline personality disorder: a multiwave study. Am J Psychiatry 2007;164(6):9228.

(18) Young JE. Cognitive therapy for personality disorders: A schema-focused approach. 3rd ed. Sarasota,FL: Professional Resources Press; 1999.

(19) Davidson K, Norrie J, Tyrer P, Gumley A, Tata P, Murray H, et al. The effectiveness of cognitive behavior therapy for borderline personality disorder: results from the borderline personality disorder study of cognitive therapy (BOSCOT) trial. J Pers Disord 2006;20(5):450-65. 
(20) Linehan MM, Comtois KA, Murray AM, Brown MZ, Gallop RJ, Heard HL, et al. Twoyear randomized controlled trial and follow-up of dialectical behavior therapy vs therapy by experts for suicidal behaviors and borderline personality disorder. Arch Gen Psychiatry 2006;63(7):757-66.

(21) Gagliesi P. Terapia Dialéctico Comportamental y la Violencia. http://www fundacionforo com/pdfs/archivo39 pdf 2006

(22) Loveland-Cherry C, Wilkerson S. Dorothy Johnson's behavioral systems model. In: J.Fitzpatrick, A.Whall, editors. Conceptual models of nursing: Analysis and application. Bowie MD; 1983.

(23) Gordon M. Manual de Diagnósticos Enfemeros. 10 ed. Madrid: Elsevier; 2003.

(24) Fornés Vives J. Enfermería de salud mental y psiquiátrica; planes de cuidados. 1 ed. Madrid: Editorial Panamericana; 2005.

(25) Heather Herdman T, Heath C, Meyer G. NANDA I: Diagnósticos enfermeros: definiciones y clasificación. Elsevier; 2007.

(26) Moorhead S, Johnson M, Maas M. Clasificación de resultados de Enfermería (NOC). $3^{\text {a }}$ ed. Elsevier; 2005.

(27) McCloskey Dochterman J, Bulechek G. Clasificación de Intervenciones de Enfermería (NIC). 4aㅡ ed. Elsevier; 2004.

(28) Treloar AJC, Lewis AJ. Targeted clinical education for staff attitudes towards deliberate self-harm in borderline personality disorder: randomized controlled trial. AUST NZ J PSYCHIATRY 2008;42(11):981-8.

(29) Ma W, Shih F, Hsiao S, Shih S, Hayter M. 'Caring across thorns' -- different care outcomes for borderline personality disorder patients in Taiwan. J CLIN NURS 2009;18(3):440-50.

(30) Bland AR, Rossen EK. Clinical supervision of nurses working with patients with borderline personality disorder. ISSUES MENT HEALTH NURS 2005;26(5):507-17. 